\title{
Eating disorders as an alternative to sexuality - how does eating disorders affect sexuality?
}

\begin{abstract}
The interest of this article aims at a possible reading on how to approach the subject of sexuality and eating disorders without necessarily talking about the body. We have tried to bring an analysis on the relation between eating disorders and sexual functioning. Indeed, we observe that the various eating disorders present an altered and/or pathological physiological, emotional and behavioral sexual functioning. How is sexuality experienced by people with these conditions? Although each individual's journey is unique and the experiences associated with eating disorders are also different, certain sexualand relational difficulties are often omnipresent. Understanding the role of eating disorders on sexual functioning and intimate relationships is essential to the development of effective therapeutic interventions for eating disorders.
\end{abstract}

Keywords: eating disorders, sexuality, anorexia, binge eating, ARFID, emotional, physiological

\author{
Volume I5 Issue I - 2022 \\ Carine Duray-Parmentier,' Noémie Nielens, ${ }^{2}$ \\ Pascal Janne, ${ }^{3}$ Maximilien Gourdin ${ }^{4,5}$ \\ 'Family and systemic psychotherapist, Psychotherapy Center of \\ Namur, Belgium \\ ${ }^{2}$ Department of Psychologist, Psychotherapy Center of Namur, \\ Belgium \\ ${ }^{3}$ Department of Psychology, Catholic University of Louvain, \\ Belgium \\ ${ }^{4}$ Department of Anesthesiology, Catholic University of Louvain, \\ Belgium \\ ${ }^{5}$ Department of Medicine, Catholic University of Louvain, \\ Belgium
}

\begin{abstract}
Correspondence: Duray-Parmentier C, Family and systemic psychotherapist, Psychotraumatologist, psychotherapy Center of Namur, rue des Fillets, 25 - 5020 Namur, Belgium,
\end{abstract} Tel 0032 4964 I I 184, Email carine.durayduray@gmail.com

Received: January 13, 2021 | Published: February 22, 2022

\section{Introduction}

The body, this "human box", a human machine so complex through which we live a panoply ofemotions that we are aware of and express through our feelings and behaviors, would be the element that would unite food and sexuality. Our relationship with our body conditions our relationship with others and inversely. Defining a healthy and balanced sexuality can be difficult for everyone, whether or not one suffers from an eating disorder. However, many people, both women and men, with an eating disorder have a disturbed body image and an altered and often negative perception of how others perceive them: a distorted body image (dysmorphophobia), low self-esteem, perfectionism and shame, which can also be an obstacle to a healthy and fulfilling sexuality. Indeed, it is simply impossible to discuss sexuality or eating disorders without referring to the human body. But how is sexuality experienced, perceived andmetabolized psychologically by people with an eating disorder? Although each individual's journey is unique and the experience associated with eating disorders is also different, certain sexual and relational difficulties are nevertheless often omnipresent. It is now recognized in thescientific literature that eating disorders can have important consequences on the lives of thosewho suffer from them. We observe a range of different mental psychopathologies associated with eating disorders.

\section{Methodology}

\section{Aims and objectives}

In this article, we have chosen to focus on the links that exist between eating disorders and intimate and sexual relationship disorders. Indeed, few scientific articles have examined the problems caused by eating disorders on the sexuality of individuals. Therefore, we will start the subject on an analytical rather than empirical level in order to provide a reflection by asking thequestion "How can eating disorders affect sexuality in individuals?" Then, we will develop, in the different sections, the psychological problems that the subject, suffering from eating disorders, would face in terms of sexuality? From the issue of dysfunctional sexuality to sexualappetite, we will compare the physiological, emotional and behavioural effects of eating disorders on sexual functioning in order to find ways for the individual to regain his or her bodythrough sexuality. Finally, we will discuss the concomitant and subsequent psychological and relational goals during the recovery process, which would lead the subjects to be able to talk about sex. Finally, the conclusion will probably allow us to understand the impact of the role of eating disorders on the sexual functioning of individuals, as the Eating Disorder could influence the development of effective interventions for eating disorders, without forgetting thepossible consequences on sexuality.

\section{The various eating disorders}

A study published in the International Journal of Eating Disorders suggests that eating disorderscan affect sexual functioning. ${ }^{1}$ In the USA alone, approximately twenty-four million peoplesuffer from eating disorders. The number of hospitalizations due to these disorders has reportedly increased significantly over the past decade. ${ }^{2,3}$ According to the Diagnostic and Statistical Manual of Mental Disorders, ${ }^{4}$ eating disorders include anorexia nervosa, bulimia nervosa, orthorexia, binge eating disorder and also newly included in the DSM-5, ARFID (Avoidant Restrictive Food Intale Disorder). Among mental health practitioners, several hypotheses are agreed upon around the idea that eating disorders are an extreme form of control that the subject exercises over his or her body, over his or her weight, through a dysfunctional relationship with food: anorexia, where the person will reduce the quantity of food as much aspossible in a mental state of deprivation, blockage, and food rejection. Bulimia is characterized by episodes of overeating and uncontrollable esophageal purging. Orthorexia is characterized by an obsession with a healthy, balanced diet and quality foods. Binge eating disorder occurs when recurrent episodes of binge eating are not associated with compensatory behaviors (vomiting, laxatives,...). ARFID is characterized by a lack of interest in eating or an avoidanceof certain foods due to their 
sensory characteristics resulting in a deficit of nutritional intake. ${ }^{5}$ As a result, people with these eating disorders are often overweight or underweight. $^{6}$ Although these eating disorders are characterized by distinct differences, they are all associatedwith an extremely negative body image perception (mental distortions) as well as an obsession with appearance. ${ }^{2}$ What about intimate relationships and sexuality when the body is continually displeased? The search for control is at the heart of eating disorders. More precisely,the subject suffering from an eating disorder thinks he/she has control over his/her appearanceand weight in an obsessive way, to relieve him/herself and/or to reassure him/herself. Often, eating disorders are associated and consequential to a PTSD (Post Traumatic Stress Disorder).Paradoxically, the subject traps himself by reinforcing himself in his vicious circle and in a low self-esteem. In intimacy, these individuals are not comfortable with the vulnerability they faceby being "laid bare" in front of another person, sharing intimate relationships and bodies with another person. When a person has an eating disorder, it creeps into both their body and their mind. It speaks to him or her as if he or she is an aggressor who is attacking his or her body andhaving intrusive negative thoughts. Faced with the intrusion, the subject develops his own personality within the person and lies constantly minimizing what is happening in his environment and in his body. This is an adaptive strategy of a mental survival state where the person dissociates and cuts himself off from his body. The subject may sacrifice himself to over-adapt to the needs of the other, while cutting himself off in order to protect himself (self-sabotage). It is often recorded that subjects suffering from eating disorders can manifest disorders of vaginismus or hypersexualization where the subject exercises an excessive sexual activity by creating a false self to adapt mentally to this game of unbalancing and unhealthy psychic dissociation for his mental balance. The eating disorders forces the individual to consider their expectations based on what others think, believe or want for them. The individual has higher standards for themselves than for others, and this revolves primarily around food, body shape and size (lack of self-indulgence, critical self-deprecation). This takeover or loss of rational and lucid control maintained by the eating disorder risks creating a "false-self" of the person: a copied, non-authentic psychic identity of the person, according to what the subject thinks in the outside world. What she wants others to see. ${ }^{7}$ Sexuality requires a "letting go" which can seem daunting for subjects suffering from eating disorders, the risk being to connectto one's true sensory self by letting go and thus being bound to one's sensory world which is tooapprehensive, feared. The disclosure of oneself to a partner is not or almost not possible because it is the feeling of loss of control that takes over. If we add to this the history of sexualabuse or incest, specialists agree that it is this feeling of loss of control, by letting the other person enter into intimacy, by surrendering to the other, that the victims do not want to reproduce. The subjects have traumatic reliviscences that the eating disorders allows the subject to think that he is camouflaging and thus exerting control over his psychic suffering through his body. As these people have been powerless in the face of their socio-familial environment, their antecedents and significant life events, they try, from then on, to regain control over themselves through their bodies, notably their weight. This would have consequences on their sexuality due to posttraumatic mental distortions.

\section{Psychological and relational problems faced by people with eating disorders in relation to sexuality?}

If there is a history of sexual trauma, this is an issue that needs to be addressed in both anorexicand overweight individuals. According to Persson, "It is important to explore the sexual history and the relationship history. If a person has not overcome the trauma, he or she will end up reliving It". Anorexic patients tend to have a distorted image of their body (dysmorphophobia). The anorexic tells herself that she is ugly, that she is disgusting, unworthyof esteeming herself because she has often been affected physically by certain traumas. The eating disorder answers that she is too large, too voluminous and that she should not eat. These are adaptive psychological processes for mental survival caused by the trauma, the mental block. The trap is to self-sabotage by maintaining automatic negative thoughts underpinned by a cognitive system with negative feedback loops. There may also be other psychological problems to overcome, such as self-esteem issues, anxiety or depression. Eating disorders are part of the short, medium- and long-term health consequences of violence and trauma experienced in childhood, adolescence or adulthood. They are associated with the presence of psychotraumatic disorders. Any life event is potentially experienced as disturbing, significantly disrupting. In fact, eating disorders would be the expression of a traumatic memory or survivalstrategy to anaesthetize the suffering and escape the trauma. The links between violence, psychotrauma and eating disorders as well as obesity are well documented in the international scientific literature, especially with sexual violence and abuse. ${ }^{9}$ Knowing the essential linksand modes of relational attachment allows us to better understand and take care of individuals suffering from eating disorders, and to finally do them justice by recognizing the traumatic origin of their suffering, as a consequence of violence committed most often by a loved one with the conscious or unconscious will to undermine and/or disrespect their physical and mentalintegrity as well as their dignity. Eating disorders are not just self-destructive behaviors or dueto a lack of willpower or psychiatric disorders. They are psychotraumatic symptoms and desperate attempts, in the greatest solitude, to find protective solutions to move forward step by step despite deep invisible wounds, and unspeakable and uncontrollable suffering that no one imagines or understands. ${ }^{10}$ Some individuals will modify their physical appearance in order to erase themselves as much as possible by a bodily negligence, an unattractive physique and/or a way to take up as little space as possible (anorexia) and to no longer be sexually attractive. It is a form of carapace. The hypothesis often encountered in psychotherapeutic follow-up with patients suffering from eating disorders is that food control is an attempt to psychically protect oneself by thinking that one is no longer a potential target or prey. The idea,here, conveyed by the subjects suffering from eating disorders, is to act unconsciously against oneself by thinking of acting for oneself in repulsion of oneself; one maintains the others at a distance and one avoids a risk of connection and sensory intrusion and of a post-traumatic reliving. According to Murielle Salmona, the lack of knowledge of many health professionals about the relationship between violence, psychotrauma and eating disorders, as well as the reality and extent of this violence and its psychotraumatic consequences, is detrimental to patients suffering from these disorders. It represents, for them, an important loss of chance and a risk of aggravating their suffering and their psychopathological degradation by exposing them to maltreatment and/or institutional professional therapeutic blunders within the framework ofcare. Indeed, the only symptomatic treatment of eating disorders is often doomed to failure: diets, nutritional follow-ups, hospitalizations and repeated relapses are likely to occur as long as the psychotraumatic origin of the eating disorders, the suffering linked to the traumatic memory, is not identified, analyzed, put into sense and treated in psychotraumatology. Indeed, the treatment of traumatic memory is essentially psychotherapeutic and essential for the person to act on his or her mental distortions. It involves its integration into an explicit narrative memory: an autobiographical, conscious and controllable memory that will no longer have colonizing power and will no longer be traumatic. ${ }^{11}$ It 
therefore requires psycho-educationalwork with the patient and his or her family to inform and explain the psychotraumatic mechanisms and the functioning of the traumatic memory. The treatment must be multidisciplinary and holistic (psychotherapy, somatic, medical, psycho-corporal, social and legal if necessary). As the traumatic memory is treated with care and meaning, the survival strategies gradually disappear. Mental patterns are psychically reworked (CBT, EMDR, EFT, Hypnosis, psychoanalysis, etc.) and negative feedback loops are gradually de-automated. The survival strategies are less and less necessary. New schemas adjusted with hindsight by the subject allow him/her to feel the trauma differently because he/she looks at it with a different reading. He can gradually overcome a state of mental survival by adjusting his mental patternsand perceptions, his distorted personal beliefs. In addition, neurological repair with neurogenscan take place, allowing the patient-victims to recover their cognitive and emotional skills, and to find or discover a feeling of coherence and unity, and to rediscover themselves. ${ }^{12}$

\section{A dysfunctional sexuality}

Subjects with eating disorders become completely disconnected from their own bodies (psychic dissociation). The disgust they feel towards themselves can only lead to an almost absolute avoidance of adequate and fulfilling intimacy. The lack of self-esteem and a constant dissatisfaction with one's body will make nudity almost absent, avoided or trivialized because it is minimized, not considered. Sexual relations will often take place in the dark with clothes that do not let the figure show. These people will also have control over the sexual positions they will choose according to what they believe to be the "least worst" for the unveiling of theirbody. It's the same for men. More and more men are also suffering from eating disorders and itis essential to talk about it, as the taboo often pushes them into a silence that is harmful to their health. ${ }^{13}$ For fear of exposing their bodily imperfections, these people may choose to keep some clothes on and negotiate a degree of nudity. Others may even feel uncomfortable performing certain positions that they feel are less advantageous and flattering. Although livinga truly fulfilling sexuality does not mean that one must necessarily achieve every sexual fantasy.

It is nevertheless important to understand that mental and emotional letting go is an essential ingredient.... to the question of sexual appetite?

In fact, desire often becomes absent or simply tries to be avoided or, as in hyper sexualization, desire is dissociated and robotized. Indeed, patients with an eating disorder may associate a lackof desire with some form of regaining control of themselves and their bodies. If they are unableto enter into a relationship with their partner (and face some difficulties), a more individualisticsexuality may be desired as a means of maintaining self-protection. The subject would rather live in thoughts through different fantasies and desires while remaining disconnected from theother. Consciously or not, the couple's sexuality would then become much more controlled andless spontaneous. It is the spouse who must then often initiate sexual relations while dependingon the preferences (in terms of sexual behaviour or frequency of relations) of the person concerned. Although sexual assault, as discussed above, is not a systematic cause of eating disorders, it can be a risk factor for them. For a victim of sexual assault, repressing desire can become a way of protecting him or herself from future assaults. Not wanting to be the object ofdesire provides a certain amount of security. By maintaining control over her appearance or body image, the victim believes she is protecting herself. Thus, people who have been victims of such aggression may end up rejecting anything feminine or sexual in their physical appearance: their breasts, buttocks, hips, thighs. ${ }^{1,11}$

\section{Physiological, emotional, and behavioral effects of eating disorders on sexual functioning}

In an effort to understand the relationship between eating disorders and sexual functioning, wewill examine the physiological, emotional, and behavioral effects on sexual functioning in individuals with eating disorders:

\section{Physiological effects}

Individuals suffering from eating disorders often report a lack of libido, symptoms of vaginismus, a condition involving a painful and unconscious tightening of the vagina, which may contribute to an inability to be able to have sexual intercourse and reach orgasm. Women with anorexia are thought to be particularly vulnerable to vaginismus because low body weight impairs the physiological functioning of the sexual organs. ${ }^{1,14}$ By restricting their diet, these anorexic women would also tend to have lower levels of cervical lubrication than those suffering from bulimia or binge eating, which means that these people with anorexia would have more pain during intercourse and difficulty reaching orgasm. As a result, they would experience less sexual satisfaction than healthy people. ${ }^{6,15-17}$ Overall, these negative physiological effects on sexual functioning, such as vaginismus and lack of libido, may prevent these individuals with eating disorders from pursuing sexual activity and, therefore, from experiencing sufficient sexual satisfaction. ${ }^{1}$

\section{Emotional effects}

Studies have shown that weight and nutrition have a significant impact on a person's interest, so that a healthy weight may promote a healthy libido. Although researchers are not sufficientlycertain of the causes of this correlation, the results would show that individuals with eating disorders would have particularly disrupted sexual interests compared to individuals without eating disorders. ${ }^{1}$ Individuals with eating disorders (anorexia, bulimia, and others) are reported to have particularly low sexual desire and more symptoms of depression, anxiety, anger and disillusionment. In addition, people who binge eat are often so insecure about their ability to control themselves that they project a sense of shame about having any sexual interest or, conversely, guilt about not wanting to have sex. Individuals with bulimia may have brief moments of sexual impulsivity, but would also generally lack stabilized sexual interest in daily life. ${ }^{1,17}$ Because individuals with eating disorders have disrupted sexual interests, they would engage in less frequent or less consistent sexual behavior. This would be one of the behavioral effects of eating disorders. ${ }^{16}$ It also appears that anorexia nervosa compared to bulimia nervosa or binge eating disorder in the individual, would present higher levels of sexual anxiety in the anorexic person as they would not feel as sexually competent. This stress could then manifest itself somatically: for example, anorexic women report experiencing unpleasant sensations because of their "anxiety". This sexual anxiety could therefore prevent subjects witheating disorders from being interested in sex..$^{14,17,18}$

\section{Behavioral effects}

According to Morgan et al, individuals with eating disorders often have fewer "normative" sexual experiences than healthy individuals. ${ }^{17}$ Indeed, according to an observation by Wiederman, female subjects with an eating disorders would have sex $80 \%$ less often than the "normal" female population. ${ }^{18}$ In contrast, compared to women with anorexia nervosa, thosewith bulimia nervosa or binge eating disorder would have more atypical sexual experiences, and at a younger age, 
according to Haimes. ${ }^{14}$ This difference could be explained by the fact that female individuals with bulimia or binge eating disorder would be more impulsive, whichwould be associated with hypersexuality and therefore registered in the adoption of unprotectedsexual behaviors with strangers with, as a consequence, difficulties in maintaining balanced sexual partners and healthy romantic relationships. ${ }^{1,6,19}$ Not only would individuals with eating disorders have fewer mentally healthy sexual partners, but they would also have fewer sexual relationships. Individuals with anorexia would have the fewest sexual partners or relationships compared to individuals with bulimic disorder or binge eating disorder. However, people with bulimia nervosa who have sexual partners would experience more tension, instability and less harmonious intimacy in their relationships. In addition, women with bulimianervosa who are reportedly married are more likely to divorce compared to healthy women. ${ }^{1,6,15,17}$ Overall, the existing literature would suggest that the most common set of eating disorders would be associated with less healthy sexual relationships. ${ }^{20}$ Furthermore, sexual behavior also includes behavior with oneself, as well as with partners. According to Calogero and Morgan, individuals with an eating disorder would report a lower frequency of masturbation than women without the disorder. In fact, women with anorexia would be the leastlikely to masturbate compared to those with bulimia or binge eating disorder. ${ }^{17,21}$ It wouldseem, according to the researchers, that women with anorexia may abstain from masturbation because they would tend to deprive themselves of any form of personal pleasure, such as foodand sex, in order to punish or preserve themselves through deprivation, self-forgetfulness. ${ }^{17}$ In contrast, women with bulimia would be twice as likely to masturbate and achieve orgasm through masturbation because they would not deny themselves the right to feel pleasure. Finally, subjects suffering from eating disorders (anorexia, bulimia and others) would not shownormative sexual self-pleasuring behavior compared to healthy subjects. ${ }^{15,17,21}$

\section{Reclaiming the body through sexuality}

Feeling calm, free of desire and impulses, in tune with one's body is possible at any age. Learning to relate to one's body, in the image it reflects, in its capacity to give and receive pleasure is an arduous path for people who have experienced trauma and have found control strategies in eating disorders. It is a whole work of psychoeducation that will have to be put in place through the different therapies. Readjusting the perception and mental distortions of one's body image, restructuring the psycho-education of healthy and balanced sexuality by learning to inhabit one's body, to love it for the (good) sensations it provides, getting into the habit of thinking and metabolizing one's body, not only with one's head, daring to ask for what brings pleasure, expressing one's desire in a straightforward and gentle way with oneself, allows the person to regain control of his or her own sexuality in a different way, and thus to discover thebenefits differently. We must not lose hope, the eating disorders can be treated and wanting to recover a fulfilled sexuality while progressing towards recovery is learning to accept oneself with one's sensoriality, to authorize oneself to feel desirable and desiring and this requires therapeutic care, patience and time. It is now appropriate to address the question of the relationship between sexuality and the body. Indeed, sexuality can reveal to us better than otherexperiences the close relationship between body, individuality and intentionality. It can also allow us to work on the bodily paradox, expressed by the proposition: "We are our body whilehaving it", within "our most intense relations with the other". ${ }^{22}$ However, we must first undertake a conceptual analysis in order to identify the use made of concepts such as sexual desire, arousal, love and pleasure, and then their meaning. According to the reductionist position of Kinsey et al..$^{23}$ arousal, pleasure and sexual desire represent simple bodily statesthat can be analyzed according to scientific criteria. In particular, arousal is analyzed as a state of physiological tension that is extinguished in the sexual act. Pleasure is described as a set of pleasant sensations related to the stimulation of the sexual organs. Sexual desire, finally, is analyzed as the desire to feel sensations of pleasure. The description of sexual life is thus no different from a description of the pleasures of the table, and the object of desire is essentially indifferent. ${ }^{24}$

\section{Concurrent and subsequent psychological and relational goals during the recovery process that lead to subjects being able to talk about "sex."}

Helping patients feel safe mentally and physically in the treatment process is paramount. Withsafety comes trust. Trust in the therapist and motivation to recover promote healing. This is called the therapeutic alliance. As this professional alliance develops, it may be possible to include the opportunity to talk about sexual needs and desires. Weight restoration and symptomimprovement or reduction must come first. Thus, there are many concurrent and subsequent psychological and relational goals during the recovery process that lead to talking about $\operatorname{sex}^{25}$

a. To help the patient to re-establish a relationship of trust and to begin to trust himself: his reactions, his thoughts, his feelings and his internal remedies, his intuitions;

b. To help the patient to feel the emotions, sometimes deeply painful or ambivalent, to bring a mental clarification and an emotional appeasement;

c. Helping the patient to rebalance his or her relationship with food, restructuring his or her mental food patterns and allowing the pathological relationship with food, rituals and obsessions with body image to be abandoned in exchange for relationships with people;

d. Helping the patient find and experience joy and pleasure in life through pleasurable sensations(for some people, this may be a first-time experience of pleasure). Gradually exposing the person to connect to their sensations and develop a secure and self-respecting sensory experience.

e. Help the patient to consider food as a pleasure of life and learn to restore a balanced and respectful relationship between consumption choices and the body, through conscious food hygiene.

It is crucial for the therapist to take the time to listen to patients talk about new activities that are only meant to provide pleasure, without compensatory behaviors related to the eating disorder (to cancel or punish the person who has indulged).

\section{Body awareness: eating pleasure versus sexual pleasure}

It is undeniable that sex is beneficial to morale and mental wellbeing, as is eating. Helping the patient to work on emotional and physical intimacy in their relationships is an important and vital goal. Generally, when a patient is well into recovery and the therapeutic alliance is deeplyestablished and solidified with the therapist, it is possible to have frank conversations about sexuality. As patients recover, they become increasingly attentive to their behavior and reactions, notice how they feel, and determine what they want, including what they want froma sexual relationship: to have a full life, including the possibility of a full and normal sex life. This is possible and is often desired by many patients. Much depends on 
the psychological stability and the degree of resilience of the subject to open up psychically to another relationshipthat may already exist through the therapeutic helping relationship so that the psychological and relational problems related to sexuality can be discovered. Food, like sex, is a pleasure. Pleasure and, in particular, orgasm, have several effects that can contribute to good psychological health: antidepressant, tranquilizer. This is mainly due to the action of the different hormones produced during the sexual act. In the same way, the physical contact, the caresses and the desire expressed during the sexual act contribute to a feeling of liberation andgeneral well-being. A fulfilled sexuality also allows to improve the self-confidence and the image that one has of the body. Having sexual relations is reassuring and allows one to be aware of the desire that one arouses and also to appropriate one's body image through the pleasant sensations experienced. Finally, by giving pleasure to his or her partner, the subject also contributes to his or her own: the fact that he or she can make someone happy also puts him or her in good mental and sensory conditions. In addition to the benefits on the psychological level, sex also has virtues on health. Indeed, regular and healthy sexual activity allows the person to be physically active and to stay in shape. Sexual activity (with a partner) can also berecommended if the person wants to lose weight or watch his or her caloric balance without getting back into the problems of eating disorders. Ten minutes of sex can burn up to 50 calories, and this by combining the useful with the pleasant. As with sports, sex, especially when practiced at a pace that suits each individual, also has a liberating power. It is an excellentway to let off steam and to evacuate toxins. Finally, the endorphins released during sex also provide a feeling of physical relaxation. Not only does sex keep you healthy, but it also has other important mental health benefits: it helps combat migraines and stress; it helps reduce therisk of cancer; it improves sleep and memory. ${ }^{26}$ Sex allows one to learn to fully inhabit one'sbody and to respond to the negative alternatives of other disorders in response to old traumas.

\section{Conclusion}

Eating disorders have negative effects on both the mental health and social life of the sufferer.Sexuality is not spared. Indeed, sexuality requires a certain approach to the body. We have attempted to provide an analysis of the relationship between eating disorders and sexual functioning. Indeed, we observe that the different eating disorders present an altered and/or pathological physiological, emotional and behavioral sexual functioning. . $^{17,18}$ Indeed, we have observed that women with eating disorders have more sexual dysfunction such as decreased vaginal lubrication and libido, as well as greater sexual anxiety and dissatisfaction than "normal" subjects. ${ }^{17,18}$ Thus, understanding the role of eating disorders on individuals' sexual functioning is critical because it could influence the development of effective eating disorder interventions. Future research should also examine other possible factors such as personality characteristics, culture of family of origin, and generational history of sexual abuse, which may mediate the relationship between eating disorders and sexual functioning. ${ }^{18}$ In addition, they should also focus more on the potential influence of cultural norms on the relationship between eating disorders and sexual functioning, as the sexual functioning of individuals from different cultures, conceptualizations, or geographic regions may be affected differently due to differing cultural views on gender. ${ }^{21}$ Secondly, we also tried to bring a reflection on the benefits of sexuality in the resumption of control of this one in the subjects suffering from eating disorders through a process of repair and psychic restructuring. Not onlywould having sexual relations be reassuring and would lead to the psycho-educational learning of being aware of the desire that the individual arouses, but they would also allow the appropriation of the body image of new sensations and a psychic rebalancing between the mindand the body. Could we, therefore, heal the body other than through eating disorders by appropriating it again in sexuality?

\section{Acknowledgments}

None.

\section{Conflicts of interest}

The authors declare that they have no conflicts of interest.

\section{Funding}

None.

\section{References}

1. Pinheiro PA, Raney TJ, Thornton ML, et al. Sexual functioningin women with eating disorders. Int J Eat Disord. 2010;43(2):123-129.

2. von Hausswolff-Juhlin Y, Brooks SJ, Larsson M. The neurobiology of eating disorders: A clinical perspective. Acta Psychiatrica Scandinavica. 2015;131(4):244-255.

3. Zhao Y, Encinosa W. Hospitalizations for eating disorders from 1999 to 2006. USA: Agency for Healthcare Research and Quality; 2006.

4. American Psychiatric Association; Diagnostic and statistical manual of mental disorders $5^{\text {th }}$ edn. Arlington, VA: American Psychiatric Publishing; 2013 .

5. Feuillet F, Bocquer A, Briend A, et al. Nutritional risk of Arfid (avoidant restrictive food intake disorders) and related behavior. Arch Pediatr. 2019;26(7):437-441.

6. Castellini G, Mannucci E, Mazzei C, et al. Sexual function in obese women with and without binge eating disorder. $J$ Sex Med. 2010;7(12):3969-3978.

7. Newton U. Intimacy and Anorexia Nervosa. 2021.

8. https://www.everydayhealth.scom/eating-disorders/how-eatingdisorders-affect-your-sex-life.aspx

9. Brewerton TD. The links between PTDS and eating Disorders. Psychiatric Times. 2003;25(6).

10. Smink FR, van Hoeken D, Hoek HW. Epidemiology, course, and outcome of eating disorders. Curr Opin Psychiatry. 2013;26(6):543-548.

11. https://gams.be/wp-content/uploads/2016/04/6_Salmona_FR.pdf

12. Ehking T, Nijenhuis ERS, Krikke A. Volume of discrete brain structures in complex dissociative disorders: preliminary findings. Prog Brain Res. 2008; 167:307-310

13. https://fr.sputniiknews.com/20190404/troubles-alimentation-anorexieprix-1040610950.html

14. Haimes AL, Katz JL. Sexual and social maturity versus social conformity in restricting anorectic, bulimic, and borderline women. International Journal of Eating Disorders.1988;7(3):331-341.

15. Abraham S. Sexuality and reproduction in Bulimia Nervosa patients over 10 years. J Psychosom Res. 1998;44(3-4):491-502.

16. Castellini G, Lorenzo L, Lo Sauro C, et al. Anorectic and Bulimic patients suffer from relevant sexual dysfunctions. J Sex Med. 2012;9(10):25902599

17. Morgan CD, Wierderman MW, Pryor TL. Sexual functionning and attitudes of eating-disordered women: A follow-up study. J Sex Marital Ther. 1995;21(2):67-77.

18. Wiederman MW, Pryor T, Lorgan CD. The sexual experience of women diagnosed with anorexia nervosa or bulimia nervosa. Int $J$ Eat Disord. 1996;19(2):109-118. 
19. Ghizzani A, Monntomoli M. Anorexia nervosa and sexuality in women: A review.

20. Journal of Sex Education \& Therapy. 2000;25(1):80-90.

21. Vaz-LealF J, Salcedo MS. Sexual adjustement and eating disorders: Differences betweentypical and atypical anorexie adolescent females. International Journal of EatingDisorders. 1991;12(1):11-19.

22. Calogero RM, Thompson K. Sexuel self-esteem in American and British college women: Relations with self-objectification and eating problems. SexRoles. 2008;60(3):160-173.

23. Nozick R. The examined life: philosophical Meditationns. $18^{\text {th }}$ edn. USA Harvard; 1989.
24. Kinsey AC, Pomeroy WB, Martin CE. Sexual Behaviour in the Human Male. CA, USA. W.B. Saunders Company; 1948.

25. Marzano M. Corps, sexuality and ethics. Thinking the body, under the direction of Marzano Michela. France: Presses Universitaires de France; 2002, pp. 83-115.

26. Scheel Judy LCSW. Sexual Satisfaction and Eating Disorders. Why weight restoration is only the first step toward sexual satisfaction. Psychologytoday. 2014.

27. https://www.passeportsante.net/sexualite-g159/Fiche.aspx?doc $=$ sexebienfaits-sexualite 